MOURA, W.M.; LIMA, P.C.; CASALI, V.W.D.; PEREIRA, P.R.G.; CRUZ, C.D. Eficiência nutricional para fósforo em linhagens de pimentão. Horticultura Brasileira, Brasília, v. 19, n. 3, p.174-180, novembro 2.001.

\title{
Eficiência nutricional para fósforo em linhagens de pimentão.
}

\author{
Waldênia M. Moura ${ }^{1}$; Paulo César Lima ${ }^{1}$; Vicente Wagner D. Casali ${ }^{2}$; Paulo Roberto G. Pereira ${ }^{2}$; \\ Cosme Damião Cruz ${ }^{2}$ \\ ${ }^{1}$ EPAMIG/ CTZM, C. Postal 216, 36.571-000, Viçosa-MG; ${ }^{2}$ UFV, 36.571-000, Viçosa-MG; Email: vwcasali@mail.ufv.br
}

\section{RESUMO}

Foram avaliadas dez linhagens de pimentão, quanto à eficiência nutricional para fósforo. $\mathrm{O}$ experimento foi conduzido em casa de vegetação, em vasos contendo $5 \mathrm{dm}^{3}$ de amostra de um latossolo com textura muito argilosa. Os tratamentos foram distribuídos em arranjo fatorial $(10 \times 5) \times 4$, sendo dez linhagens de pimentão, cinco doses de fósforo $(0 ; 250 ; 500 ; 750$ e $1.000 \mathrm{mg}$ de $\mathrm{P} / \mathrm{kg}$ de solo) e quatro repetições, com delineamento em blocos casualizados. Constatou-se ampla variabilidade genética entre as linhagens quanto à eficiência nutricional para fósforo $(\mathrm{P})$, a qual foi decorrente, principalmente das variações na eficiência de enraizamento, de absorção e de utilização do elemento para produção de matéria seca de parte aérea, uma vez que observou-se poucas variações na eficiência de translocação do P. Maiores eficiências de enraizamento não refletiram em maiores aquisições de $\mathrm{P}$ do solo, sugerindo que a absorção do elemento foi influenciada por características morfológicas e fisiológicas do sistema radicular. Dentre as linhagens estudadas a L10 foi a mais eficiente na absorção e utilização do P. Também a L8 mostrou bom comportamento. Já as linhagens L1, L2 e L6 mostraram-se menos eficientes na utilização do P. As outras linhagens apresentaram comportamentos variáveis em relação aos índices de eficiência avaliados. Essa ampla variabilidade observada pode ser explorada em programas de melhoramento genético visando maior eficiência nutricional para $\mathrm{P}$.

Palavras-chave: Capsicum annuum, fosfato, enraizamento, absorção, translocação, utilização.

\begin{abstract}
Phosphorus efficiency of sweet pepper lines.

Ten sweet pepper lines were screened for phosphorus efficiency. An experiment was carried out in the greenhouse in pots filled with $5 \mathrm{dm}^{3}$ of clayey latosol soil samples. The treatments followed a randomized complete block design, in a factorial layout $(10 \times 5 \times 4)$, comprising ten lines, five doses of $\mathrm{P}(0 ; 250 ; 500 ; 750$ and $1,000 \mathrm{mg}$ of $\mathrm{P} / \mathrm{kg}$ of soil) and four replications. Results showed genetic variability among lines for P-efficiency, characterized mainly by rooting efficiency, P-uptake and P-use efficiency. Low differences in the P- translocation efficiency were observed. Higher rooting efficiency was not translated into higher acquisition of $\mathrm{P}$ from the soil, which suggests that $\mathrm{P}$-absorption was influenced by morphologic and physiologic characteristics of the root system. The L10 line showed high P-efficiency uptake and utilization, followed by the L8 line. The L1, L2 and L6 lines, were the least efficient in the Putilization. The other lines showed variable performance in Pefficiency, according to the index used. The large variability observed might be explored in genetic improvement programs aiming to increase the nutritional efficiency for $\mathrm{P}$.
\end{abstract}

Keywords: Capsicum annuum, fosfate, rooting, uptake, translocation, utilization.

(Aceito para publicação em 14 de setembro de 2.001)

\begin{abstract}
A grande maioria dos solos brasileiros são ácidos, com baixa fertilidade e elevada capacidade de retenção de fósforo, o que leva à necessidade de aplicação de elevadas doses de fosfatos, contribuindo para o aumento nos custos de produção, além de reduzir os recursos naturais não renováveis que originam esses insumos. Assim, tem-se intensificado a busca para o aproveitamento do potencial adaptativo de genótipos às condições adversas de fertilidade do solo, por meio do melhoramento genético, ou seja, cultivares que apresentam maiores eficiência nutricional.

Inúmeros conceitos de eficiência nutricional, têm sido relatados na lite-
\end{abstract}

ratura, os quais variam com o nutriente e a espécie de planta (Gerloff, 1976; Whiteaker et al., 1976; Siddiqi \& Glass, 1981; Coltman et al., 1985; Bailian et al., 1991). Tal variação está sendo amplamente questionada pois dificulta uma conceituação geral, bem como a comparação dos resultados. A eficiência nutricional pode ser conceituada como a quantidade de biomassa produzida por unidade do nutriente aplicado no solo, que depende de dois componentes principais (Bailian et al., 1991): a eficiência de aquisição e a eficiência de utilização. O primeiro componente, depende das eficiências de absorção (parâmetros cinéticos) e de enraizamento (parâmetros morfológicos). Já o segundo componente, depende das eficiências de translocação e de conversão em biomassa. Em se tratando do fósforo, considera-se como plantas eficientes aquelas que produzem maior quantidade de matéria seca por unidade de fósforo absorvido (Gerloff, 1976). Também, considera-se plantas eficientes aquelas que acumulam maiores concentrações de fósforo quando cultivadas em baixas doses deste elemento (Clark \& Brown, 1974), ou que atingem maiores produções por unidade de tempo e área (Fox, 1978). Já, Föhse et al. (1988) definiram a eficiência para fósforo como sendo a 
habilidade das plantas em produzir certa percentagem de produção máxima com certa dose de fósforo no solo. Portanto, a eficiência para fósforo, seja em baixa disponibilidade, ou em suprimento adequado do elemento, relaciona-se com a maior produção de biomassa, associada ao menor consumo de fósforo. Estudos baseados nesses conceitos têm demonstrado que tanto plantas bem supridas como deficientes em $\mathrm{P}$ podem apresentar alta taxa de eficiência. Assim, para evitar a seleção de plantas eficientes na utilização de P, porém com baixa produção, Siddiqi \& Glass (1981) propuseram o índice de eficiência: (matéria seca produzida $)^{2} /$ unidade do nutriente absorvido, reunindo num mesmo índice a eficiência de utilização do nutriente e o crescimento.

Existem numerosas evidências de variabilidade genética em diferentes espécies de plantas quanto à absorção, translocação, acúmulo e à utilização de P (Whiteaker et al., 1976; Coltman et al., 1985; Buso \& Bliss, 1988). Essas variações constituem a base genética para programas de melhoramento. Entretanto um dos aspectos críticos na maioria desses programas, é a identificação dos mecanismos responsáveis por tais variações. Sob baixa disponibilidade de $\mathrm{P}$, várias alterações morfológicas, fisiológicas e, ou, bioquímicas do sistema radicular podem favorecer a absorção do elemento, como: desenvolvimento de raízes laterais (Gerloff \& Gabelman, 1983) e de pêlos radiculares, (Föhse \& Jungk, 1983), redução no diâmetro de raízes e aumento da superficie de raízes (Schenk \& Barber, 1979; Föhse \& Jungk, 1983), aumento na velocidade máxima de absorção (Martinez et al., 1993b), aumento no influxo de P e redução do Km (Coltman et al., 1985; Martinez et al., 1993a). Também, as associações micorrízicas (Marschner, 1986), mudanças no $\mathrm{pH}$ da rizosfera, exsudação de compostos orgânicos pelas raízes e níveis de fosfatase nas células das raízes (Clarkson, 1985), podem influenciar a absorção de fósforo.

Uma vez que o nutriente é absorvido, o movimento do íon através das raízes e sua liberação para o xilema envolvem vários passos, que podem limitar sua liberação para a parte aérea das plantas, podendo ser a base das diferenças genotípicas na absorção e no movimento dos nutrientes (Gerloff \& Gabelman, 1983). Comumente, plantas deficientes em fósforo retêm mais $\mathrm{P}$ nas raízes e o translocam menos para a parte aérea, em comparação com plantas bem-supridas (Bieleski, 1973; Martinez et al, 1993b). Em virtude da retenção de $\mathrm{P}$ nas raízes, o crescimento radicular é mantido em detrimento do desenvolvimento da parte aérea, aumentando a relação raiz/parte aérea, que é uma característica que pode estar associada com a deficiência de fósforo. $\mathrm{O}$ transporte de $\mathrm{P}$ para a parte aérea foi a característica mais importante na diferenciação entre genótipos de capim-colonião cultivado sob estresse de P (Furlani \& Usberti Filho, 1990), ao contrário do observado para alface (Buso \& Bliss, 1988) e soja (Martinez et al., 1993b). Existem grandes variações no transporte de $\mathrm{P}$ das raízes para a parte aérea entre as espécies monocotiledôneas e as dicotiledôneas, pois as primeiras são mais dependentes da via metabólica para o transporte de $\mathrm{P}$, que consiste em três etapas principais: esterificação do Pinorgânico, logo após a entrada nas raízes; utilização do P-orgânico no metabolismo das raízes; hidrólise do P-orgânico e liberação do P-inorgânico para o xilema (Loughman, 1978).

O fósforo translocado é utilizado no metabolismo e crescimento. Vários fatores afetam a eficiência de utilização do $\mathrm{P}$, como a distribuição do nutriente na planta, que é influenciada pela intensidade de retranslocação e reutilização, como observado em sorgo (Wieneke, 1990), trigo (Horst et al., 1993) e cevada (Raju et al., 1987). Também a incapacidade da planta em liberar o fósforo inorgânico $(\mathrm{Pi})$ do vacúolo quando o estresse se desenvolve após um período de suprimento adequado de fósforo, pode resultar na menor eficiência de utilização desse elemento (Bieleski, 1973). A concentração de Pi nos tecidos da planta interfere em vários processos metabólicos, dentre eles a fotossíntese. Baixas concentrações de Pi no citossol promovem a redução da fotossíntese, por reduzirem a atividade de várias enzimas do ciclo de Calvin, principalmente a atividade da ribulose-5-fosfato cinase, e consequentemente a regeneração da ribulose bisfosfato (RuBP) (Rao \& Terry, 1989). Por outro lado, altas concentrações de Pi também reduzem a fotossíntese, pois a exportação excessiva de trioses-P para o citossol influencia a regeneração da RuBP, que é o aceptor de $\mathrm{CO}_{2}$ (Morison \& Batten, 1986). A maior eficiência de utilização de $P$ entre linhagens de feijão cultivadas sob estresse de $\mathrm{P}$ foi associada com a maior taxa fotossintética líquida (Whiteaker et al., 1976).

Portanto, vários mecanismos contribuem, para as diferenças entre genótipos, quanto à eficiência nutricional para fósforo. O conhecimento desses permitiria uma seleção mais efetiva, contribuindo para o avanço dos programas de melhoramento genético.

Neste trabalho, procurou-se identificar fontes de variabilidade genética e avaliar mecanismos envolvidos na eficiência nutricional para fósforo em dez linhagens de pimentão.

\section{MATERIAL E MÉTODOS}

O experimento foi realizado em casa de vegetação do Departamento de Fitotecnia da Universidade Federal de Viçosa. O material vegetal utilizado originou-se do cruzamento entre dois híbridos: (BGH 4562 x BGH 4561) e (BGH 18 x BGH 2665). O híbrido duplo obtido foi posteriormente cruzado com um terceiro híbrido (BGH $18 \mathrm{x}$ BGH 2854), obtendo-se 200 linhagens, as quais foram avançadas até as gerações $\mathrm{F}_{12}$, sem seleção artificial. Em função da quantidade de sementes disponível, selecionaram-se 10 linhagens para este estudo. Os tratamentos foram distribuídos em blocos casualizados, num arranjo fatorial $10 \times 5$ (dez linhagens $\mathrm{x}$ cinco doses de $\mathrm{P}$ ), com quatro repetições, totalizando-se 200 unidades experimentais. Utilizou-se um solo com baixo teor de fósforo, classificado como LU (Latossolo variação UNA), do Município de Viçosa-MG. A amostra do solo foi coletada na camada subsuperficial (0 - $20 \mathrm{~cm}$ ), seca ao ar, destorroada, passada em peneira com abertura de $4 \mathrm{~mm}$ e homogeneizada. Subamostras foram utilizadas para caracterizações química e física, apresentando $\mathrm{pH} \mathrm{em} \mathrm{H}_{2} \mathrm{O}(1: 2,5)$ 


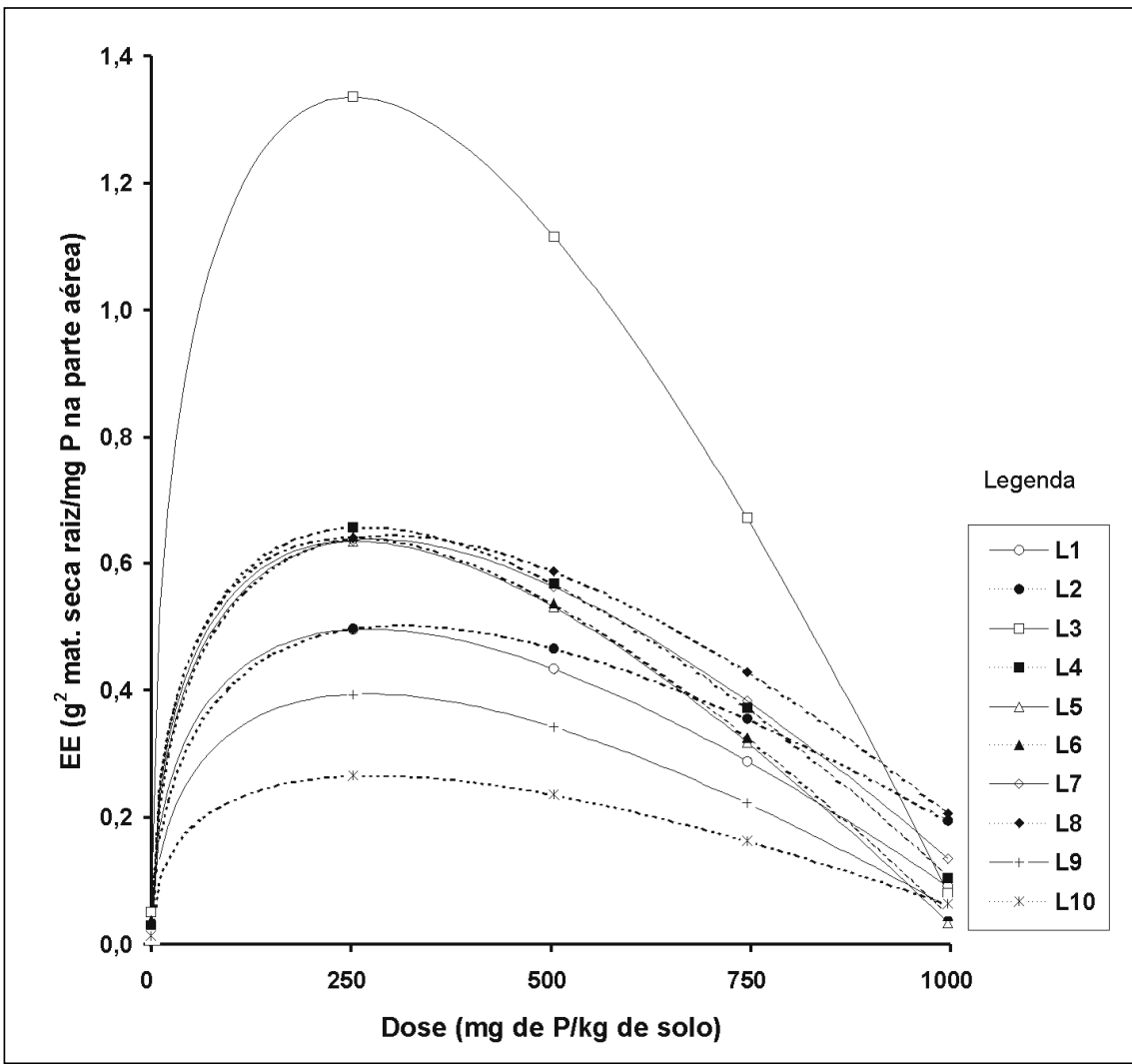

Figura 1. Eficiência de enraizamento (EE) de 10 linhagens de pimentão, em cinco doses de fósforo. Viçosa, UFV, 1993/94.

de 5,$1 ; 1,5 \mathrm{mg}$ de $\mathrm{P} / \mathrm{dm}^{3}$ e $10 \mathrm{mg}$ de $\mathrm{K} /$ $\mathrm{dm}^{3}$ (Extrator Mehlich-1, Vettori, 1969); 0,1 cmolc de $\mathrm{Ca}^{2+} / \mathrm{dm}^{3} ; 0,0$ cmolc de $\mathrm{Mg}^{2+} / \mathrm{dm}^{3}$ e $0,7 \mathrm{cmolc}^{\mathrm{de}} \mathrm{Al}^{3+} / \mathrm{dm}^{3}$ (Extrator KCl mol/L,Vettori, 1969); 14\% de areia grossa; $14 \%$ de areia fina, $3 \%$ de silte, $69 \%$ de argila (método da pipeta, EMBRAPA,1979); Capacidade de campo de 33\% (Método da coluna transparente, Fernandes \& Sykes, 1968) e classificação textural, como muito argiloso.

A acidez do solo foi corrigida, fazendo-se a calagem de acordo com as recomendações da Comissão de Fertilizantes do Solo do Estado de Minas Gerais (1989) e a aplicação de calcário na dose correspondente a 3,19 t/ ha, com relação $\mathrm{Ca}: \mathrm{Mg}$ de 4:1, em equivalente. Posteriormente, adicionou-se $1 \mathrm{cmolc} /$ $\mathrm{dm}^{3}$ de $\mathrm{Mg}\left(\mathrm{MgCl}_{2} \cdot 6 \mathrm{H}_{2} \mathrm{O}\right)$, em coberturas parceladas em cinco aplicações semanais, reduzindo a relação $\mathrm{Ca}: \mathrm{Mg}$ para 2:1. Quinze dias após a calagem, adicionou-se o fósforo nas doses de $0 ; 250$; 500; 750 e $1.000 \mathrm{mg}$ de $\mathrm{P} / \mathrm{kg}$ de solo, fornecidas numa combinação dos seguintes sais puro para análise: $\left(\mathrm{NH}_{4}\right) \cdot \mathrm{H}_{2} \mathrm{PO}_{4}, \mathrm{KH}_{2} \mathrm{PO}_{4}, \mathrm{NaH}_{2} \mathrm{PO}_{4}$ e $\mathrm{Ca}$ $\left(\mathrm{H}_{2} \mathrm{PO}_{4}\right) \cdot \mathrm{H}_{2} \mathrm{O}$, baseado no peso atômico
$1,56\left(\mathrm{FeSO}_{4} \cdot 7 \mathrm{H}_{2} \mathrm{O}\right) ;$ Mo $-0,15$ $\left(\mathrm{NaMo}_{4} \cdot 2 \mathrm{H}_{2} \mathrm{O}\right) ; \mathrm{B}-0,18\left(\mathrm{H}_{3} \mathrm{BO}_{3}\right)$ e $\mathrm{Mn}$ - 3,66 $\left(\mathrm{MnCl}_{2} \cdot 4 \mathrm{H}_{2} \mathrm{O}\right)$. Quando as plântulas apresentaram duas folhas definitivas, realizou-se o desbaste deixando uma planta por vaso. Durante a condução do experimento, o solo foi mantido com a umidade próxima da capacidade de campo. As plantas foram conduzidas tutoradas e as capinas foram manuais. $\mathrm{O}$ experimento foi colhido aos 120 dias após a semeadura, quando as plantas foram seccionadas rente ao solo. O material vegetal da parte aérea foi separado em caule, folha e fruto e o sistema radicular foi retirado dos vasos com o auxílio de jato d'água dirigido sobre o substrato. Todos os órgãos da planta foram acondicionados em sacos de papel e secos a $75^{\circ} \mathrm{C}$ em estufa com circulação de ar forçada até peso constante. Após a secagem, determinou-se o peso da matéria seca de raiz e parte aérea (caule, folha e fruto). Adicionouse ao peso da matéria seca de fruto, os frutos colhidos durante todo o período experimental. Posteriormente, a matéria seca foi moída em moinho Willey, com peneira no 20 , homogeneizada, sendo dela retiradas amostras equivalentes a $2 \mathrm{~g}$, as quais foram submetidas à digestão nitroperclórica; no extrato obtido, foram dosados os teores de P (Braga $\&$ Defelipo, 1974). Os conteúdos de fósforos foram obtidos pelo produto entre os teores de $\mathrm{P}$ e a matéria seca de cada órgão da planta. Uma vez obtidos esses dados, estimaram-se os seguintes índices de eficiência, baseado na proposta de Sidiqui \& Glass, 1985: 1) Eficiência de enraizamento (EE): ( $\mathrm{g}$ de matéria seca de raiz)2/mg de $\mathrm{P}$ na parte aérea; 2 ) Eficiência de absorção de P (EAP): mg de $\mathrm{P}$ na parte aérea/g de matéria seca de raiz. 3) Eficiência de translocação de $P$ (ETP): $\mathrm{mg}$ de $\mathrm{P}$ na parte aérea/mg de $\mathrm{P}$ total. e 4) Eficiência de utilização do fósforo na parte aérea (EUPPA): ( $\mathrm{g}$ de matéria seca da parte aérea $)^{2} / \mathrm{mg}$ de $\mathrm{P}$ na parte aérea.

Os dados obtidos para cada variável foram submetidos à análise de variância, e as fontes de variação consideradas foram blocos, linhagens, doses de fósforo e a interação linhagem $x$ dose de fósforo. Foram ajustadas equações de regressão, em função das doses de fósforo, 
Tabela 1. Equações e coeficientes de regressão para dez linhagens de pimentão para a eficiência de enraizamento (EE), eficiência de absorção de fósforo (EAP), eficiência de translocação de fósforo (ETP) e eficiência de utilização de fósforo na parte aérea (EUPPA), em função de cinco doses de P adicionadas ao solo. Viçosa, UFV, 1993/94.

\begin{tabular}{|c|c|c|c|c|}
\hline $\begin{array}{l}\text { Linha- } \\
\text { gens }\end{array}$ & EE & $\mathbf{R}^{2}(\%)$ & EAP & $\mathbf{R}^{2}(\%)$ \\
\hline L1 & $\ddot{Y}=0,0114+0,0588^{\star \star} \sqrt{X}-0,0018^{\star *} X$ & 88 & $\ddot{Y}=-0,0295+0,0321^{* *} X$ & 91 \\
\hline L2 & $\ddot{Y}=-0,000003+0,06^{* *} \sqrt{ } X-0,002^{* *} X$ & 77 & $\ddot{Y}=0,7236+0,0269 * * X$ & 80 \\
\hline L3 & 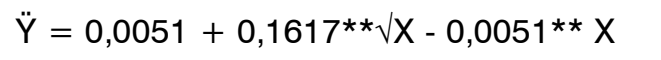 & 84 & $\ddot{Y}=-1,0713+0,0242^{* *} X$ & 95 \\
\hline L4 & $\ddot{Y}=0,0297+0,0771^{\star \star} \sqrt{X}-0,0024^{\star *} X$ & 76 & $\ddot{Y}=0,259+0,0214^{* *} X+0,000009^{* *} X 2$ & 99 \\
\hline L5 & $\ddot{Y}=0,0095+0,0785^{\star \star} \sqrt{X}-0,0025^{\star *} X$ & 88 & $\ddot{Y}=0,0331-0,542^{\star \star} \sqrt{X}+0,0553^{* *} X$ & 90 \\
\hline L6 & $\ddot{Y}=0,0376+0,0763^{\star \star} \sqrt{X}-0,0024^{\star *} X$ & 70 & $\ddot{Y}=-0,3263+0,0312^{\star *} X$ & 94 \\
\hline L7 & $\ddot{Y}=0,0034+0,0760^{\star \star} \sqrt{X}-0,0023^{\star *} X$ & 94 & $\ddot{Y}=0,87+0,0153^{\star *} X+0,000016^{* *} X 2$ & 96 \\
\hline L8 & $\ddot{Y}=0,0021+0,0743^{\star *} \sqrt{X}-0,0022^{* *} X$ & 99 & $\ddot{Y}=0,2784+0,0248^{* *} X$ & 96 \\
\hline L9 & $\ddot{Y}=-0,0065+0,0486^{\star *} \sqrt{ } X-0,0015^{\star *} X$ & 87 & $\ddot{Y}=1,4114+0,0179^{* *} X+0,00002^{* *} X 2$ & 96 \\
\hline L10 & $\ddot{Y}=0,0137+0,0304^{* *} \mathrm{O} X-0,0009^{* *} X$ & 72 & $\ddot{Y}=0,6775+0,0405^{\star *} X$ & 88 \\
\hline $\begin{array}{l}\text { Linha- } \\
\text { gens }\end{array}$ & ETP & $\mathbf{R}^{2}(\%)$ & 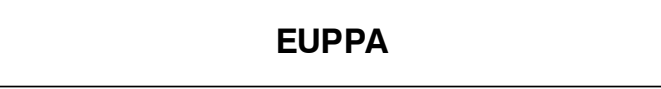 & $\mathbf{R}^{2}(\%)$ \\
\hline L1 & $\ddot{Y}=0,0093+0,0737^{* *} \mathrm{O} X-0,0014^{* *} X$ & 99 & $\ddot{Y}=0,1067+4,4888^{* *} \sqrt{X}-0,1309^{* * X}$ & 84 \\
\hline L2 & $\ddot{Y}=0,01001+0,0727^{\star \star} \sqrt{X}-0,0014^{\star *} X$ & 98 & $\ddot{Y}=0,7368+4,3823^{\star \star} \sqrt{X}-0,1293^{\star *} X$ & 85 \\
\hline L3 & $\ddot{Y}=0,0086+0,0703^{\star \star} \sqrt{X}-0,0013^{\star *} X$ & 99 & $\ddot{Y}=0,5335+4,5609^{\star *} \sqrt{X}-0,1314^{\star *} X$ & 86 \\
\hline L4 & $\ddot{Y}=0,0075+0,0731^{\star \star} \sqrt{X}-0,0014^{\star *} X$ & 99 & $\ddot{Y}=1,0620+5,2687^{\star \star} \sqrt{X}-0,1565^{\star \star} X$ & 88 \\
\hline L5 & $\ddot{Y}=0,0088+0,0719^{\star *} \sqrt{X}-0,0013^{* *} X$ & 99 & $\ddot{Y}=1,7089+5,0238^{* *} \sqrt{X}-0,1519^{* *} X$ & 81 \\
\hline L6 & $\ddot{Y}=0,0066+0,0725^{\star \star} \sqrt{X}-0,0014^{\star *} X$ & 99 & $\ddot{Y}=0,5545+4,3031 * * \sqrt{X}-0,1239^{* *} X$ & 92 \\
\hline L7 & $\ddot{Y}=0,0109+0,0712^{\star \star} \sqrt{X}-0,0013^{\star *} X$ & 98 & $\ddot{Y}=0,7651+5,0881^{* *} \sqrt{X}-0,1443^{* *} X$ & 89 \\
\hline L8 & $\ddot{Y}=0,0096+0,0716^{\star \star} \sqrt{X}-0,0014^{\star \star} X$ & 98 & $\ddot{Y}=1,1499+6,2862^{\star \star} \sqrt{X}-0,1895^{\star \star} X$ & 85 \\
\hline L9 & $\ddot{Y}=-0,0112+0,0753^{\star \star} \sqrt{X}-0,0015^{\star \star} X$ & 98 & $\ddot{Y}=1,1883+4,9554^{\star *} \sqrt{X}-0,1477^{\star *} X$ & 87 \\
\hline L10 & $\ddot{Y}=0,0078+0,0756^{\star \star} \sqrt{X}-0,0015^{\star *} X$ & 99 & $\ddot{Y}=1,4528+6,1718^{\star \star} \sqrt{X}-0,18^{\star \star} X$ & 88 \\
\hline
\end{tabular}

** - Significativo a nível de $1 \%$ de probabilidade pelo teste $\mathrm{t}$.

como variável independente e, como dependentes, as variáveis avaliadas, nas linhagens. Testaram-se vários modelos de equações, selecionando as de maiores coeficientes de determinação ajustados e significativos até o nível de 5\% de probabilidade.

\section{RESULTADOS E DISCUSSÃO}

Eficiência de enraizamento (EE)

Todas as linhagens apresentaram respostas com ajuste ao Modelo Raiz Quadrada, diante da aplicação de P no solo, embora tenham mostrado diferentes magnitudes de eficiência (Tabela 1). Em geral, observou-se resposta crescente para todas as linhagens até a dose 250 $\mathrm{mg}$ de $\mathrm{P} / \mathrm{kg}$ de solo. À medida que adicionou-se $\mathrm{P}$ ao solo, ocorreu uma redução na EE (Figura 1).

A linhagem L3 foi a mais eficiente, ou seja, investiu na maior produção de matéria seca do sistema radicular por unidade do $\mathrm{P}$ absorvido, enquanto as linhagens L10 e L9 foram as menos eficientes. As outras linhagens apresentaram comportamento intermediário entre as citadas acima. A eficiência de enraizamento foi um dos mecanismos responsáveis pela maior eficiência nutricional de plantas de soja cultivadas em solução nutritiva, sob baixa disponibilidade de P (Martinez et al., 1993b). Entretanto, nesta condição o fósforo encontra-se prontamente disponível para as plantas, o que não ocorre quando utiliza-se o solo como substrato no plantio. Contudo, vale ressaltar que a eficiência de enraizamento, refletida pela produção de matéria seca de raiz, torna-se importante desde que esteja associada a uma alta eficiência de absorção de fósforo, em decorrência da baixa mobilidade do $\mathrm{P}$ no solo. (EAP)

\section{Eficiência de absorção do fósforo}

As linhagens apresentaram marcantes aumentos da EAP, com o fornecimento de P (Figura 2). Dentre as linhagens estudadas a L10 destacou-se pela maior EAP, exceto na dose de 1.000 $\mathrm{mg}$ de $\mathrm{P} / \mathrm{kg}$ de solo. Nas doses mais elevadas de $\mathrm{P}$ (750 e $1.000 \mathrm{mg}$ de $\mathrm{P} / \mathrm{kg}$ de solo), além da L10, as linhagens L5 e L9 também apresentaram altas EAP, enquanto que as linhagens L3 e L8, apresentaram menores EAP. Observou-se também que, a linhagem L10, mais eficiente na absorção de $\mathrm{P}$, apresentou menor EE (figura 1). Tal fato indica que, apesar da menor produção de matéria seca de raiz por unidade de $\mathrm{P}$ absorvido, possui um sistema radicular bastante eficiente na aquisição de $\mathrm{P}$ do solo. Por outro lado, as linhagens L3 e L8, menos eficiente em absorver $\mathrm{P}$, exibiram altos valores para $\mathrm{EE}$, sugerindo 


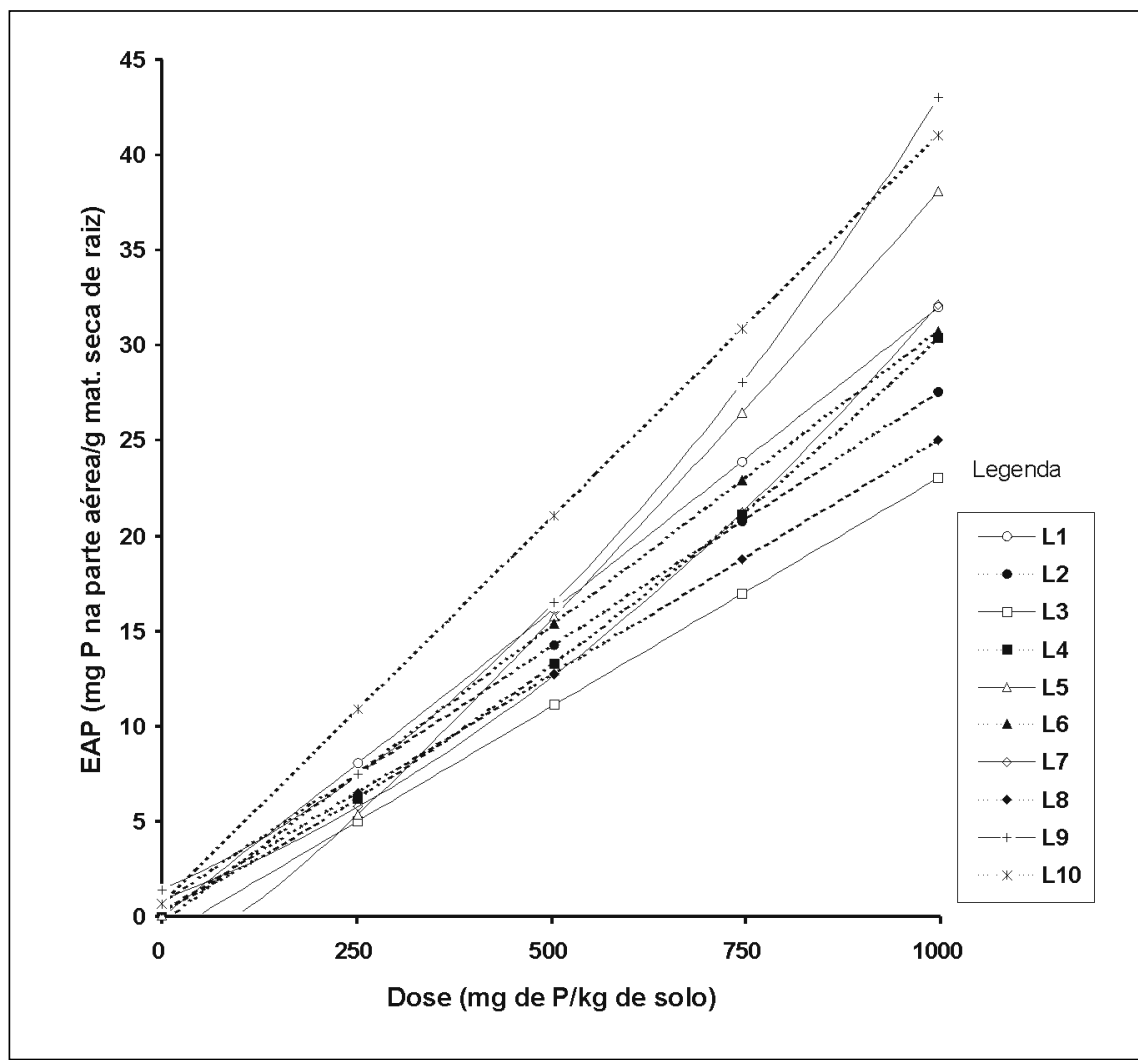

Figura 2. Eficiência de absorção de fósforo (EAP) de 10 linhagens de pimentão, em cinco doses de P. Viçosa, UFV, 1993/94.

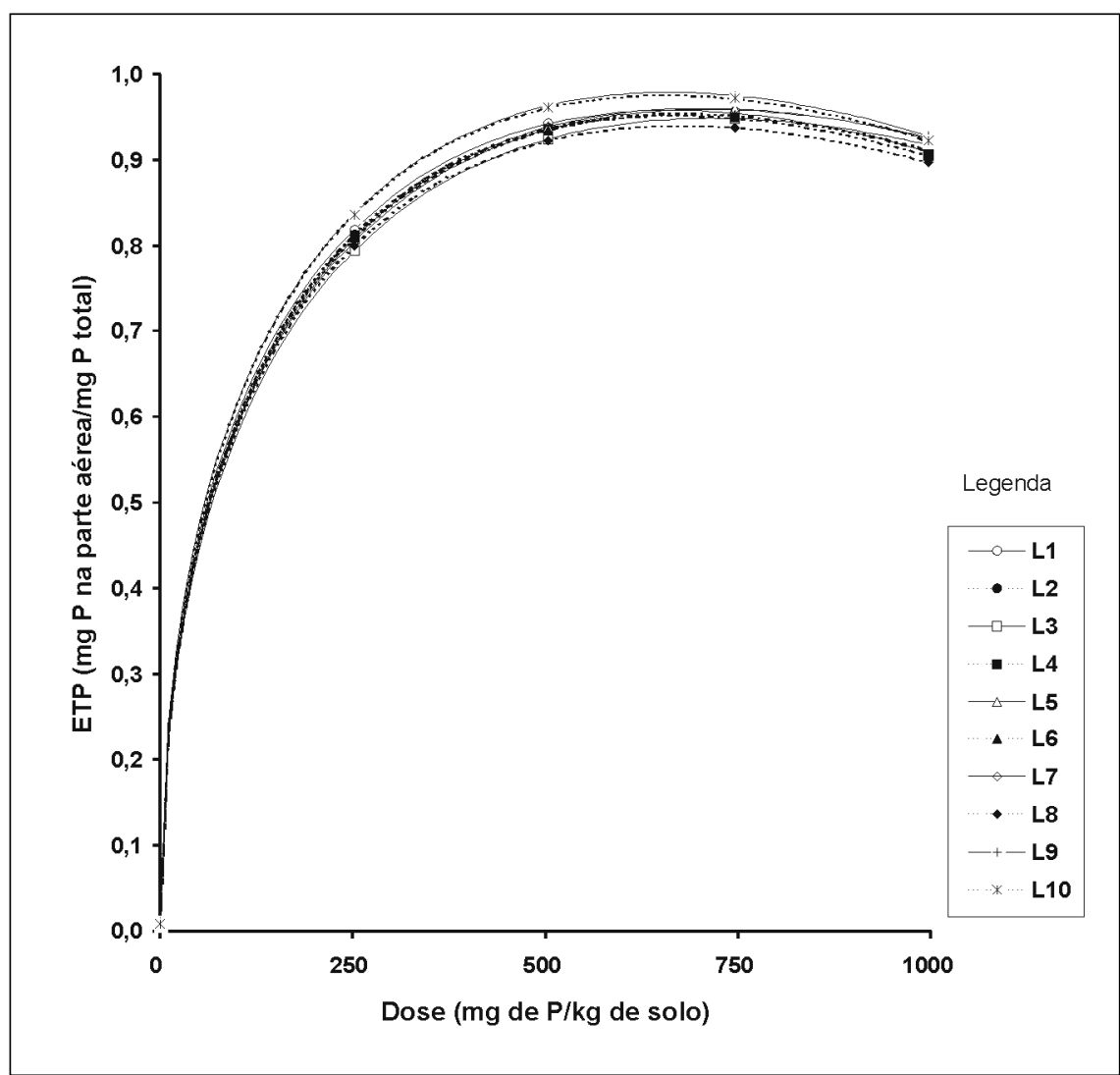

Figura 3. Eficiência de translocação de fósforo (ETP) de 10 linhagens de pimentão, em cinco doses de P. Viçosa, UFV, 1993/94. sistemas radiculares pouco efetivos na aquisição de $\mathrm{P}$ do solo. Diferenças na eficiência de absorção entre genótipos de cevada foram associadas às variações no comprimento radicular e nas taxas de absorção de $\mathrm{P}$ por unidade de comprimento de raiz (Schjorring \& Nielsen, 1987). Em trigo, a maior eficiência de absorção de P da cultivar Coris foi relacionada com o menor diâmetro radicular e a presença de pêlos radiculares (Horst et al., 1993). Diferenças observadas no influxo de $\mathrm{P}$ entre várias espécies de plantas foram decorrentes das diferenças de Vmax, além do número e comprimento de pêlos radiculares, que podem ser responsáveis por $90 \%$ da absorção total do nutriente (Föehse et al., 1991). Esses resultados reforçam o fato que, além da matéria seca de raiz produzida, outras características como a morfologia e os parâmetros cinéticos de absorção, foram fundamentais para a aquisição desse nutriente. Entretanto, existem dificuldades de avaliar esses parâmetros quando utiliza-se o solo como substrato.

Eficiência de translocação do fósforo (ETP)

As linhagens apresentaram respostas com ajustes ao Modelo Raiz Quadrada (Tabela 1), e apresentaram comportamentos similares (Figura 3). Esses resultados indicaram que a ETP pode ser um mecanismo não limitante no processo metabólico de assimilação do $\mathrm{P}$ que pouco contribuiu para a diferenciação entre as linhagens quanto à resposta a adubação fosfatada. Resultados semelhantes foram observados em alface (Buso \& Bliss, 1988) e soja (Martinez et al., 1993b), ao contrário do constatado para capim-colonião (Furlani \& Usberti Filho, 1990). Provavelmente, esse fato pode estar associado com as variações existentes do transporte de $\mathrm{P}$ das raízes para a parte aérea entre as espécies monocotiledôneas e as dicotiledôneas (Loughman, 1978).

Eficiência de utilização do fósforo na parte aérea (EUPPA)

Novamente, todas as linhagens apresentaram respostas com ajuste ao Modelo Raiz Quadrada, porém, com diferentes magnitudes (Tabela 1). A EUPPA aumentou com o suprimento de $\mathrm{P}$ até próximo de $250 \mathrm{mg}$ de P/kg de solo (Figura 4). A 
partir desta dose, com o aumento da disponibilidade de $\mathrm{P}$ no solo, a absorção do mesmo cresceu em proporções maiores que a elevação da produção de matéria seca da parte aérea, resultando em decréscimo na EUPPA. Altas concentrações de P nos tecidos podem reduzir a fotossíntese, em virtude da exportação excessiva de trioses-P do cloroplasto para o citossol, reduzindo também os intermediários do ciclo de Calvin e consequentemente a produção de fotoassimilados (Morison \& Batten, 1986).

A linhagem mais eficiente na utilização do fósforo na parte aérea foi a L10, fato que pode ser atribuído à maior intensidade de redistribuição de $\mathrm{P}$ dos tecidos velhos e inativos para os jovens em desenvolvimento, (Raju et al., 1987; Wieneke, 1990; Horst et al., 1993). Essa linhagem, também apresentou alta eficiência de absorção de P. A linhagem L8, embora não tenha se destacado com relação aos outros parâmetros avaliados, apresentou alta eficiência na utilização de P. As linhagens L1, L2 e L6 foram as menos eficientes na utilização de $\mathrm{P}$, e as outras linhagens apresentaram comportamento intermediário.

Assim, constataram-se ampla variabilidade genética entre as linhagens de pimentão estudadas, com relação à eficiência nutricional para fósforo, que pode ser atribuído principalmente às eficiências de enraizamento, de absorção e de utilização do elemento para produção de matéria seca de parte aérea, uma vez que observaram-se poucas variações na eficiência de translocação do P.

\section{AGRADECIMENTOS}

Os autores agradecem à FAPEMIG, pelo apoio financeiro à realização deste trabalho.

\section{LITERATURA CITADA}

BAILIAN, L.; McKEAND, S.E.; ALLEN, H.L. Genetic variation in nitrogen use efficiency of lobeolly pine seedlings. Forest Science, v. 37, n. 2, p. 613-626, 1991.

BIELESKI, R.L. Phosphate pools, phosphate transport, and phosphate availability. Annual Review of Plant Physiology, v. 24, p. 225-252, 1973. BRAGA, J.M.; DEFELIPO, B.V. Determinações espectrofotométricas de fósforo em extratos de solos e material vegetal. Revista Ceres, Viçosa, v. 21, p. 73-85. 1974

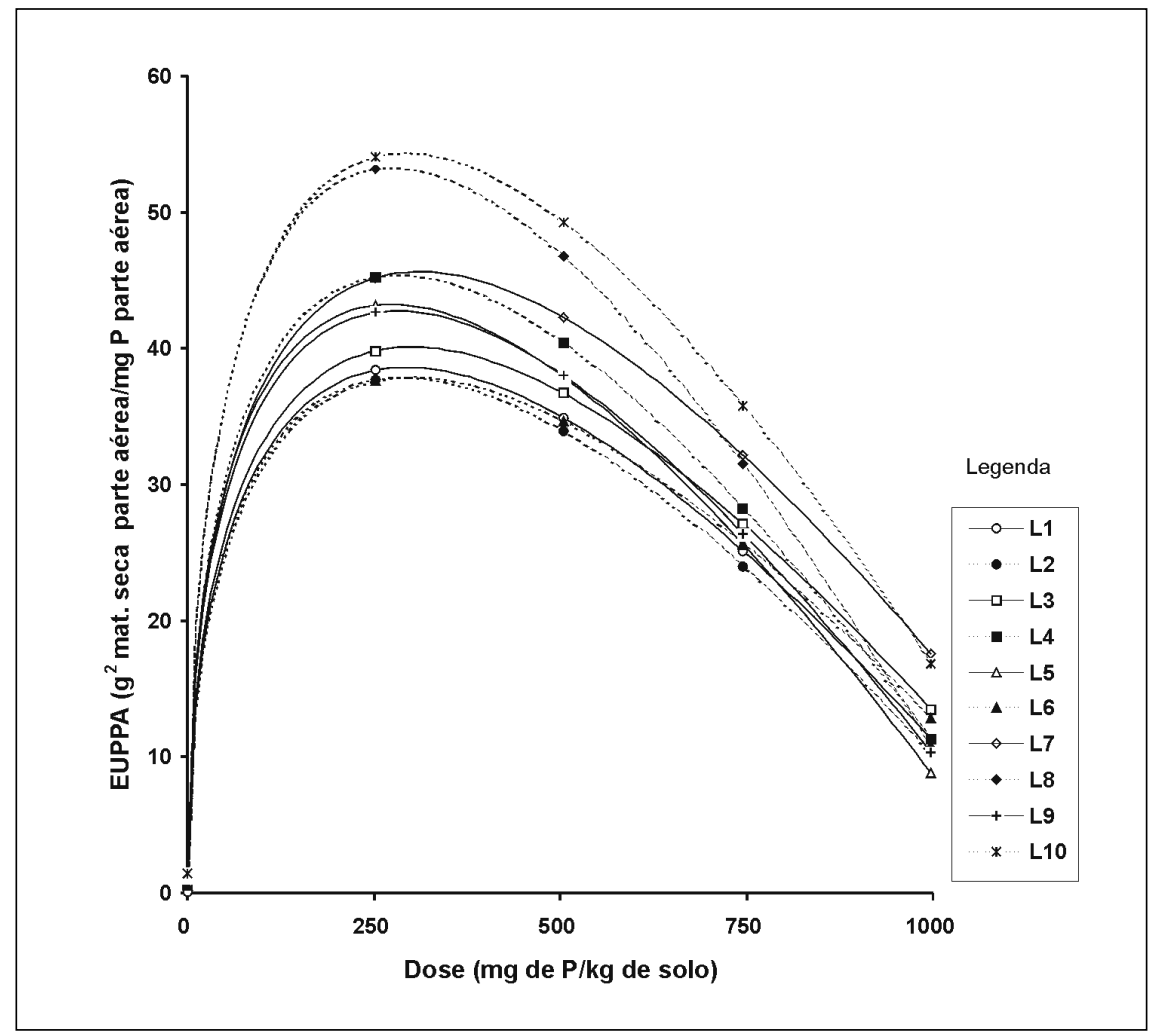

Figura 4. Eficiência de utilização de fósforo (EUPPA) de 10 linhagens de pimentão, em cinco doses de P. Viçosa, UFV, 1993/94.

BUSO, G.S.C.; BLISS, F.A. Variability among lettuce cultivars grown at two levels of available phosphorus. Plant and soil, v. 111, p. 67-73, 1988. CLARK, R.B.; BROWN, J.C. Differential phosphorus uptake by phosphorus-stressed can inbreds. Crop Science., v. 14, p. 505-508, 1974.

CLARKSON, D.T. Factors affecting mineral nutrient acquisiton by plants. Annual Review of Plant Physiology, v. 36, p. 77-115, 1985.

COLTMAN, R.R.; GERLOFF, G.C.; GABELMAN, W.H. Differential tolerance of tomato strains to maintained and deficient levels of phosphorus. Journal of the American Society of Horticultural Science, v. 110, n. 2, p. 140-144, 1985. COMISSÃO DE FERTILIDADE DO SOLO DO ESTADO DE MINAS GERAIS. Recomendações para o uso de corretivos e fertilizantes em Minas Gerais. 4. Aproximação. Lavras: CFSEMG, 1989. 176 p.

Embrapa. Serviço Nacional de Levantamento e Conservação de Solo. EMBRAPA-SNLCS. $M a$ nual de métodos de análise de solo. Rio de Janeiro, 1979. n. p.

FERNANDES, B.; SYKES, I.O. Capacidade de campo e retenção de água em três solos de Minas Gerais. Revista Ceres, Viçosa, v. 15, p. 1-39, 1968. FÖHSE, D.; CLAASSEN, N.; JUNGK, A. Phophorus efficiency of plants. I. External and internal $\mathrm{P}$ requeriment and $\mathrm{P}$ uptake efficiency of different plant species. Plant and Soil, v. 110, p. 101-109, 1988.

FÖHSE, D.; CLAASSEN, N.; JUNGK, A. Phosphorus efficiency of plants. II. Significance of root radius, root hairs and cation-anion balance for phosphorus influx in seven plant species. Plant and Soil, v. 132, p. 261-272, 1991.
FÖHSE, D.; JUNGK, A. Influence of phosphate and nitrate supply on root hair formation of rape, spinach and tomato plants. Plant and Soil, v. 74, p. 359-368, 1983.

FOX, R.H. Selection for phosphorus efficiency in corn. Communications in Soil Science and Plant Analysis, v. 9, p. 13-37, 1978.

FURLANI, A.M.C.; USBERTI FILHO, J.A. Capim-colonião: eficiência na absorção e na utilização de fósforo em solução nutritiva. Bragantia, Campinas, v. 49, n. 2, p. 413-423, 1990.

GERLOFF, G.C. Plant efficiencies in the use of nitrogen phosphorus and potassium. In: WHIGHT, M.J. (Ed.). Proceedings of workshop on plant adaptation to mineral stress in problem soils. Beltsville: Maryland, 1976. p. 161-173.

GERLOFF, G.C.; GABELMAN, W.H. Genetic basis of inorganic plant nutrition. In: LÄUCHLI, A.; BIELESKI, R.L. (Eds.). Inorganic plant nutrition. Encyclopedia of Plant Physiology, v. 15 B, Berlin: Springer- Verlag, 1983. p. 453-480.

HORST, W.J.; ABDOU, M.; WIESLER, F. Genotypic differences in phosphorus efficiency of wheat. In: BARROW, N.J. (Ed.). Plant nutrition from genetic engineering to field practice. Dordrecht: Kluwer Academic Publishers. 1993. p. 367-370.

LOUGHMAN, B.C. Metabolic factors and the utilization of phosphorus by plants. In Phosphorus in the environment: its chemistry and biochemistry. Amsterdam, Elsevier, 1978. p. 155-173.

MARSCHNER, H. Mineral nutrition of higher plants. London: Academic Press, 1986. 674 p. 
MARTINEZ, H.E.P.; NOVAIS, R.F.; RODRIGUES, L.A.; SACRAMENTO, L.V.S. Comportamento de variedades de soja cultivada em diferentes doses de fósforo: I. Cinética de absorção de fósforo e ajustes morfológicos da planta. Revista Brasileira de Ciência do Solo, Campinas, v. 17, p. 231-238, 1993a.

MARTINEZ, H.E.P.; NOVAIS, R.F.; SACRA MENTO, L.V.S.; RODRIGUES, L.A. Comportamento de variedades de soja cultivadas sob diferentes níveis de fósforo. II. Translocação do fósforo absorvido e eficiência nutricional. Revista Brasileira de Ciência do Solo, Campinas, v. 17, p. 239-244, 1993b.

MORISON, J.I.L.; BATTEN, G.D. Regulation of mesophyll photosynthesis in intact wheat leaves by cytoplasmic phosphate concentrations. Planta, v. 168 , p. 200-206, 1986.
RAJU, P.S.; CLARK, R.B.; MAITI, R.K.; MARANVILL, J.W. Phosphorus uptake, distribution and use by glossy and nonglossy sorghum. Journal of Plant Nutrution, v. 10, p. 2017-2024, 1987.

SCHENK, M.K.; BARBER, S.A. Root characteristics of corn genotypes as related to $\mathrm{P}$ uptake. Agronomy Journal, v. 71, p. 921-24, 1979. SCHJORRING, J.K.; NIELSEN, N.E. Root length and phosphorus uptake by four barley cultivars grown under moderate deficiency of phosphorus in field experiments. Journal of Plant Nutrition, v. 10, n. 9-16, p. 1289-1295, 1987.

SIDDIQI, M.Y.; GLASS, A.D.M. Utilization index: a modified approach to the estimation and comparision of nutrient utilization efficiency in plants. Journal of Plant Nutrition, v. 4, n. 3, p. 289-302, 1981.
VETTORI, L. Métodos de análise de solo. Rio de Janeiro: Ministério da Agricultura, EPF, 1969. 24 p. Boletim técnico $\mathrm{n}^{0} 7$.

WHITEAKER, G.; GERLOFF, G.C.; GABELMAN, W.H.; LINDGREN, D Intraspecific differences in growth of beans at stress levels of phosphorus. Journal of the American Society for Horticultural Science, v. 101, n. 4, p. 472-475, 1976.

WIENEKE, J. Phosphorus efficiency and phosphorus remobilization in two sorghum (Sorghum bicolor (L.) Moench) cultivars. Plant and Soil, v. 123, p. 139-145, 1990. 